\title{
Why they don't understand?
}

\section{Roberta Ceccarelli \\ Società Italiana di Urologia Oncologica (SIUrO), Bologna, Italy}

\section{Introduction}

On a daily basis, we are involved in communication processes with colleagues, family members, friends and, in health environment, with patients.

In most cases, we do not ask ourselves how these processes happen, neither we care for the effects on the person we are talking to. Usually, in fact, the consequences of what we say coincide with our expectations and, when this does not happen, we do not even try to understand why.

For example, when we give a friend directions to our home, we do expect him to arrive. If this event does not occur, it is very likely that we will draw the following conclusion: he got lost!.

In most cases, then, we do not care about the effects our words have on the others, that is to say, we do not care if our communication was effective or not.

The single biggest problem in communication is the illusion that it has taken place -

George Bernard Shaw

This article aims to show when an effective communication is performed, both in general and in the patient-physician interaction. In a brief premise, we will describe the elements of communication, then the barriers to an effective communication will be considered together with their effects. Finally, some suggestions for making our communication with patients more effective will be offered. Obviously, this is just a mention to a very complex topic, therefore references for further in-depth studies are reported.

Correspondence: Roberta Ceccarelli, Società Italiana di Urologia Oncologica (SIUrO), Bologna, Italy.

E-mail: roberta.ceccarelli67@gmail.com

Key words: effective communication, misunderstanding.

Received for publication: 25 November 2014.

Accepted for publication: 25 November 2014.

This work is licensed under a Creative Commons Attribution NonCommercial 3.0 License (CC BY-NC 3.0).

(C) Copyright R. Ceccarelli, 2014

Licensee PAGEPress, Italy

Oncology Reviews 2014; 8:273

doi:10.4081/oncol.2014.273

\section{The elements of communication}

First of all, it is important to remind that in communication processes the actors involved are generally two or more. However, in order to simplify the following discussion, we will refer only to a communication between two subjects. Therefore, there will be the sender, that is to say the person who sends the message, and the receiver, that is to say the person who receives the message (Figure 1).

These two roles will switch at every communication passage: the sender will become the receiver, and the receiver will become the sender (Figure 2).

Furthermore, human communication occurs through two channels: verbal and non-verbal. ${ }^{1}$ The first one refers to the words, the sentences and the grammatical structure we use to communicate. The second one, the non verbal channel, including the paraverbal elements of communication, refers to the body language, the facial expressions, hand movements, the pauses between words, the tone of voice, the distance from the receiver (prossemic) and so on.

In those highly emotional situations more than $90 \%$ of the message is conveyed by non-verbal language. ${ }^{2}$

A significant breakthrough in the study of communication is represented by the publication of the book Pragmatic of human communication in $1967 . .^{1}$ In fact, the authors of this volume give a completely new perspective on analyzing and studying human communication.

The first important element of change comes from considering a communication pattern as a behavior. Therefore, given that behavior has no opposite..., there is no such thing as nonbehavior (that is to say one cannot not behave), then an opposite of communication does not exist either. In other words, as stated by the first axiom of communication:

\section{one cannot not communicate.}

Doing or not doing something, speaking or staying silent, all have the capacity to express a message.

This implies that, whatever we do, our behavior/communication will always have an impact on other people, who will naturally react with another behavior/communication. Therefore it can be stated that communication is a behavior which always elicits reactions and other behaviors.

We previously saw that in any communication we activate, in parallel, the verbal and non-verbal channels. It is worth noting that, while the first one gives information on the content of the message, the second one gives fundamental information about the relationship between sender and receiver. ${ }^{1}$

If we read the following sentences:

In these cases is recommended to repeat the exams in three months

And 


\section{Do you want me to repeat the exams in three months?}

We can say that both convey the same content - to repeat an exam in three months - but we are sure that the relationship between speakers in the first sentence is different from the relationship between speakers in the second sentence, though the non-verbal part is very limited.

every communication has a content and a relationship aspect, ... the two modes of communication not only exist side by side but complement each other every message $e^{1}$

Finally, in every communication interaction, there is an aspect that is extremely important even though it is overlooked most of the time. Each actor of a communication pattern will define the communication and the complementary relationship from his/her own perspective or point of view (Figure 3).

It is as in the example given by Watzlawick of the rat who says: - I have got my experimenter trained. Each time I press the lever he gives me food.

To summarize: it is not possible not communicate: each communication interaction expresses a part of content and a part of relationship, the last is conveyed by non verbal language; each actor participates in the communication from his/her own perspective.

\section{When communication is effective?}

To talk about effective communication we need to introduce a further element: the goal of communication. ${ }^{3{ }^{4}}$ In fact, it is possible to say that a behavior was effective only in relation to its goal. Similarly, we can say that a communication was effective only in relation to the aim with which we started the communication interaction. Furthermore, the closer to our goal we are, the more effective has been our communication.

At this point it is worth noting that, given what has been told previously, it is possible for us to know if we reached our goal only from the receiver's reaction/answer.

Communication is the receiver's feedback, not the sender's intention

Taking the example that was proposed at the beginning of this article, I will know if the communication about the way to reach my house has been effective only when I see my friend at destination.

Considering physician patient communication, as reported in research, ${ }^{5,6}$ the most important goals are respectively, to give and to receive the best care available. Obviously these objectives could have several sub-objectives. On the one hand, physicians would aim at saving patients' life, helping them making decisions among the available therapies (when it is possible), informing patient on adverse factors of therapies, making patients to meet therapeutic schedule and followup, enrolling patients in clinical trials. On the other hand, patients would aim at getting rid of the problem as soon as possible, keeping their life style and their social relationship, participating at the grand son baptism, and so on. ${ }^{5}$

For both doctors and patients, the communication interaction, and the connected relationship, will result effective as far as they will be able to reach their goals. ${ }^{5,6}$ In fact, given the aspects of interaction and relationship involved in communication, even when only one of the actors is not able to reach his/her goal, communication cannot be considered effective. Let us think, for example, that during a physician patient consultation none of the patient's concerns about the schedule of the proposed therapy emerged. However, these concerns will be evident the first time the patient will miss an appointment. This does not mean that therapies have to be changed according to the patient's wish. On the contrary, this means that in order to reach our goal to let the patient meet the therapy schedule should be worthwhile giving patient time to express his/her worries and concerns and sharing the therapeutic goals..$^{5-7}$

\section{Barriers to an effective communication}

Barriers to an effective communication can have various origins. ${ }^{8,9}$ They may come from the organization in which we work as well as from the way we act in a given situation. The first case includes, for example, the unavailability of adequate spaces and settings for confidential consultation as well as a too short time for visits. Since organizational regulation and rectification of these barriers ought to be dealt separately and by other expertise, only barriers of the second case will be considered. Furthermore, depending on our attitude toward the situation, it would be possible to learn how to manage them.

Among these barriers there are: the lack of motivation for an effective communication; the use of a technical language; the habit of referring to own culture's scheme; and the rules of thumb that we use every day to manage the complexity of our world. ${ }^{9,10}$

The latters are particularly significant because we use them spon-

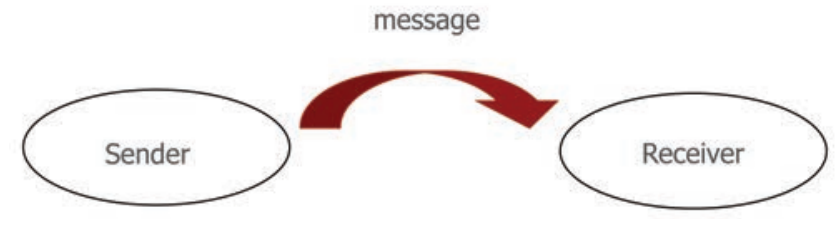

Figure 1. First passage of a communication pattern.

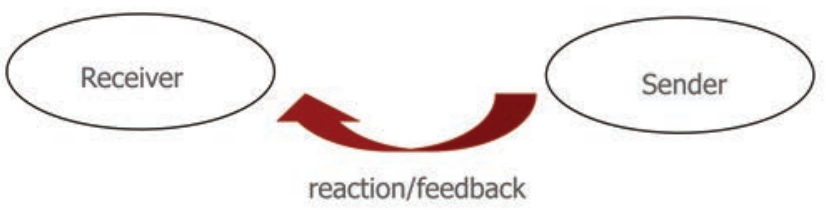

Figure 2. Second passage of a communication pattern.
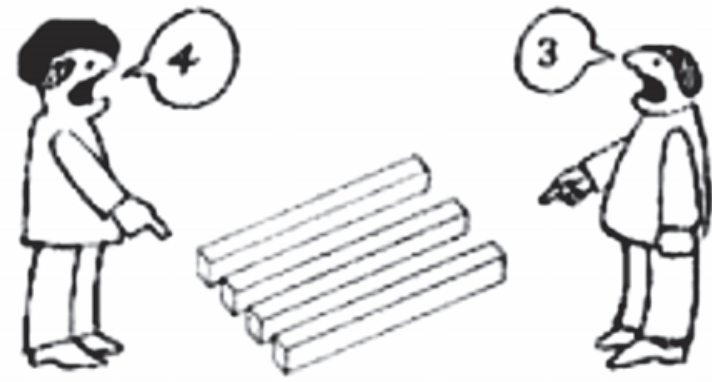

Figure 3. Each subject defines the communication from his/her point of view. 
taneously and can affect dramatically the effectiveness of our communication.

Tversky and Kahneman, in an article published in $1974^{11}$ demonstrated how people rely on a small number of cognitive strategies, defined as heuristics, which help to simplify difficult tasks such as making a decision under uncertainty. In most cases these cognitive shortcuts are very useful (otherwise we would have already discarded them) allowing us to make a decision quickly and effectively. However, in some cases, they give rise to biases and errors of judgment.

According to the representativeness heuristic, for example, an object is assigned to a certain category rather than another one, insofar as its principal characteristics are representative or recall that category, rather than the other one.

When we judge objects or events as frequent, likely or causal agents, only because they are easily available in our memory, then we are using the availability heuristic.

If we rely on the first piece of information we receive as anchor or as reference point to make a decision or a judgment, then we are using the anchoring heuristic.

What kind of effect could have these cognitive mechanisms on communication patterns?

One of the most important moments in the physician patient relationship is the collection of patient's health data by the physician. Furthermore, this information has to be collected directly from the patient. Nevertheless, research show that physicians have the tendency to interrupt patients before they had the time to express their worries and concerns. For example, Beckman and Frankel ${ }^{12}$ found that patients were interrupted, on average, 18 seconds after they have started talking. A more recent research ${ }^{13}$ shows similar results (patients were interrupted after $23 \mathrm{~s}$ on average).

Physicians who interrupt patients prematurely could rely on the representativeness heuristic. In fact, they could recognize some characteristics in the patient or in his/her story that are representative of a certain group of patients and assume the overall picture.

However, this attitude can have a great impact on patient physician communication and even more on the developing relationship.

As previously stated, each behavior communicates something. Therefore, the physician who interrupts a patient is very likely to communicate that he/she is in a hurry, that he/she is very busy or, in the worst case, that he/she is not interested in what the patient is saying (by the way, answering the cell phone during a consultation could deliver the same message).

According to the availability heuristic an event is erroneously considered more likely (or causal agent) only because it comes first to mind. ${ }^{11}$ This heuristic could give reason to the evaluation error on risks and benefits of a treatment. In fact, depending on the sequence in which they are presented, they will remain more or less impressed in memory. In other words, when the therapy benefits are presented before its risks, these last will come to mind more easily (because they are more recent). Consequently risks could also be considered more likely (because they are recalled better) and more numerous (because more of them are recalled). ${ }^{10,14-17}$

Considering the anchoring heuristic, even this can give reason of the patient's choice of therapy. For example, if the first prostate cancer therapeutic option, which is proposed by the physician, were the surgery, than its adverse effect would work as anchor for the evaluation of the other available treatments. ${ }^{16,17}$ Rather, each therapy should be evaluated separately and the impact on patient's quality of life should be taken as reference point.

To complete the picture of effective communication we need to talk about listening. Communication is a circular process, it is a repeated exchange of message/behavior and feedback. ${ }^{1}$ Consequently, in order to reach an effective communication, active listening plays a fundamental role (Figure 4).
Literature distinguishes five levels of listening: i) ignoring; ii) pretend listening; iii) selective listening; iv) attentive listening; v) empathic (or active) listening ${ }^{18}$ (Figure 5). None of the first four levels of listening guarantee an effective communication. To listen in active and emphatic way in health field, it is necessary to treat every consultation as though each problem is completely new and never heard before.

This approach involves a series of attitudes that make the patient feel really understood and welcome. Thanks to this ability, communication takes place in a situation that, on the one hand, is particularly apt to acknowledge the patient and, on the other hand, makes patient entrusting the physician.

Same attitude of active listening makes us to go beyond words, and truly understand the real feeling of the person we are talking to. Active listening means not to be limited to literal interpretation of the message but it means going further and try to understand both their statements and their behaviors. To achieve active listening, it is important to read between the lines, to focus the attention on the others, to understand the emotions behind their reports. Achieving active listening does not require a great amount of time. Instead it requires to dedicate real and sincere attention to others, accepting their point of view and their way of thinking (though it could be far from our own), showing that we are really interested in their words through our answers. ${ }^{18}$

Our skills and our effectiveness in communicating depend on our ability to detect systematically how the receiver reacts (using words and behavior) in order to constantly adjust and calibrate our communication.

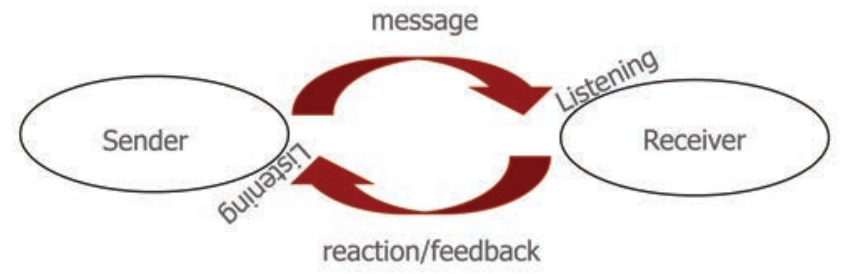

Figure 4. Importance of active listening.

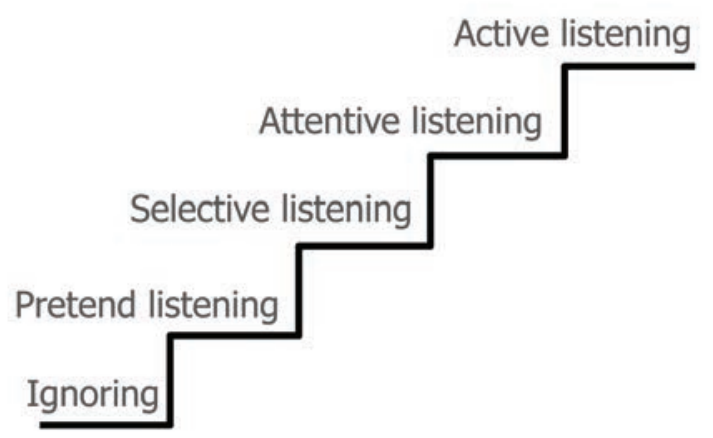

Figure 5. Five levels of listening. 


\section{Conclusions}

The problem of communication is surely larger and more complicated compared to what we have reported in this article. Its main goal, as stated at the beginning, was to draw the reader's attention to a behavior that, in most cases, is managed without problems.

Patient physician communication, however, represents a more fragile interaction compared to those that we usually encounter. In fact, especially with oncological patients, strong emotions and stress are at stake. ${ }^{19-21}$ Moreover, patients are required to make quick decisions on very important aspects of their life.

As stated before, in every communication patterns there are at least two actors and all barriers to effective communication affect both the physician and the patient. Thus, patients are victims of the same cognitive mechanisms as physicians. For example, it may happen that a patient does not pay attention, maybe just because he/she is feeling strong emotions.

However, it is worth noting that physician-patient relationship is not symmetrical (all participants are at the same level for knowledge and status). On the contrary physician-patient relationship is complementary as defined by the contest. ${ }^{1}$ In a complementary relationship one of the participant has a one up position because of a higher level of knowledge or experience in a particular field. ${ }^{1}$ From this perspective, in the physician-patient relationship, the physician is in a one up position. Thus he/she has the responsibility to conduct the communication exchange helping patients to express their concerns and trying to create a satisfying relationship through an effective communication.

Since the first axiom of communication states that one cannot not communicate, when it is particularly important to achieve an effective communication, we ought to deal with the problem of communication and we are required to formulate strategies (as how to explain risks and benefits of a particular treatment), to define objectives (sharing them with patients), to listen actively.

Leaving all these aspects to chance does not mean not communicate (that is not possible, as it has been shown). On the contrary, it means to communicate exactly what we do not want to communicate like: lack of interest, superficiality, ambiguity, uncertainty.

Therefore, it means leaving the receiver (the patient) under the uncertainty about the goals and the aims pursued by the sender (the physician), with the chance that to this last would be attributed the most absurd and contradictory objectives.

\section{References}

1. Watzlawick P, Beavin JH, Jackson DD. Pragmatic of human communication. A study of interactional patterns, pathologies, and paradoxes. New York, London: W.W. Norton \& Co.; 1967.

2. Mehrabian A. Silent messages. Belmont, CA: Wadsworth Publ. Co.;
1971.

3. Simpson M, Buckman R, Stewart M, et al. Doctor-patient communication: the Toronto consensus statement. BMJ 1991;303:1385-7.

4. Feldman-Stewarta D, Brundagea MD, Tishelmane C; SCRN Communication Team. A conceptual framework for patient professional communication: an application to the cancer context. Psychooncology 2005;14:801-9.

5. Butow P. The communication goals and needs of cancer patients: a review. Psychooncology 2005;14:846-7.

6. Buzaglo JS, Millard JL, Ridgway CG, et al. An internet method to assess cancer patient information needs and enhance doctorpatient communication: a pilot study. J Cancer Educ 2007;22:23340.

7. Lam WWt, Fielding Richard, et al. Gambling with your life: the process of breast cancer treatment decision making in chinese women. Psychooncology 2005;14:1-15.

8. Khan TM, Hassali MA, Al-Haddad MSM. Patient-physician communication barrier: a pilot study evaluating patient experiences. $\mathrm{J}$ Young Pharm 2011;3:250-5.

9. Ellis C. The doctor and medical student's perspectives of the doctor-patient relationship: an analysis of communication. Int $\mathrm{J}$ Commun Health 2013;1:16-23.

10. Hall KH. Reviewing intuitive decision-making and uncertainty: the implications for medical education. Med Educ 2002;36:216-24.

11. Tversky A, Kahneman D. Judgment under uncertainty: heuristics and biases. Sci New Ser 1974;185:1124-31.

12. Beckman HB, Frankel RM. The effect of physician behavior on the collection of data. Ann Intern Med 1984;101:692-6.

13. Marvel MK, Epstein RM, Flowers K, Beckman HB. Soliciting the patient's agenda: have we improved? JAMA 1999;281:283-7.

14. Covey J. A meta-analysis of the effects of presenting treatment benefits in different formats. Med Decis Making 2007;27:638-54.

15. Carrigan N, Gardner PH, Conner M, Maule J. The impact of structuring information in a patient decision aid. Psychol Health 2004;19:457-77.

16. Klein WMP, Stefanek ME. Cancer risk elicitation and communication: lessons from the psychology of risk perception. CA Cancer J Clin 2007;57:147-67.

17. Rothman AJ, Salovey P. Shaping perceptions to motivate healthy behavior: the role of message framing. Psychol Bull 1997;121:3-19.

18. Burley-Allen M. Listening the forgotten skill. New York, NY: John Wiley \& Sons; 1982.

19. Braddock CH, Snyder L. The doctor will see you shortly. the ethical significance of time for the patient-physician relationship. J Gen Intern Med 2005;20:1057-62.

20. Fagerlind H, Lindblad AK, Bergtrom I, et al. Patient-physician communication during oncology consultations. Psychooncology 2008;17:975-85.

21. Mauksch LB, Dugdale DC, Dodson S, Epstein R. Relationship, communication and efficiency in the medical encounter. Arch Intern Med 2008;168:1387-95. 